\title{
Profile of Apoptotic Proteins after Curcumin Treatment by Antibody Array in H69AR Lung Cancer Cells
}

\author{
Süleyman Ilhan ${ }^{1}$ \\ 'Manisa Celal Bayar University, Faculty of Science and Letters, Department of Biology, Manisa, Turkey
}

ORCID IDs of the authors: S.I. 0000-0002-6584-3979

Please cite this article as: Ilhan S. Profile of Apoptotic Proteins after Curcumin Treatment by Antibody Array in H69AR Lung Cancer Cells. Eur J Biol 2020; 79(2): 157-162. DOI: 10.26650/EurJBiol.2020.0015

\begin{abstract}
Objective: The aim of the study was to investigate the changes in the expression levels of apoptosis-related proteins after treatment with curcumin (Cur) on multiple drug-resistant H69AR non-small cell lung cancer cells.

Materials and Methods: Viability of H69AR cells after Cur exposure $(5-100 \mu \mathrm{g} / \mathrm{mL})$ was evaluated via MTT assay at 24,48 and $72 \mathrm{~h}$. Apoptosis was assessed via ELISA assay. Apoptosis related proteins of breast cancer cell lines were analyzed by a Human Apoptosis Antibody Array. Protein-protein interactions were analyzed and visualized by using the STRING database.

Results: Cur inhibited cell viability and induced apoptosis in H69AR cells. The IC50 value of Cur in H69AR cells was $8.75 \mu \mathrm{g} /$ $\mathrm{mL}$. The array results showed that the protein levels of pro-apoptotic proteins such as Bad, Bax, Caspase-3, TRAIL R1, TRAIL R2, FADD, Fas, SMAC/DIABLO, HMOX2 were significantly increased by 2.4-, 3.1-, 2.6-, 3.1-, 3.4-, 2.4-, 2.1-, 4.1- and 5.5-fold in H69AR cells $(p<0.05)$. Moreover, the protein levels of the anti-apoptotic proteins such as Bcl-2, clAP-1, CLU and HIF1A were significantly decreased by 4.1-, 3.2-, 2.2- and 2.0-fold, respectively in H69AR cells by Cur exposure ( $p<0.05)$.
\end{abstract}

Conclusion: Findings of this study suggested that Cur induced apoptosis of human H69AR cells via mediating several proteins involved in both extrinsic and intrinsic apoptotic pathways.

Keywords: Curcumin, apoptosis, protein array, protein-protein interactions

\section{INTRODUCTION}

Cancer is one of the major public health problems globally. Lung cancer is the most common cancer type and a leading cause of cancer-related deaths. Despite advances in lung cancer therapy, the mortality rate of the disease is extremely high and the 5-year survival rate is approximately $16 \%$. (1). Non-small cell lung cancer (NSCLC) is the most common type, which composes of nearly $85 \%$ of all cases. Toxic effects and various side effects limit the efficiency of standard therapy for lung cancer (2). Moreover, drug resistance that leads to tumor recurrence and disease progression is the major problem for therapeutic failure (3). Therefore, there is an urgent need for novel alternative agents to inhibit proliferation and to induce apoptosis of multiple drug-resistant (MDR) lung cancer cells.
Natural plant products are a valuable source of novel bioactive compounds having potential cytotoxic activities. Curcumin (Cur) is a polyphenolic compound originated from the plant Curcuma longa L. called turmeric (4) and was extracted from the rhizomes of this plant in a pure crystalline form in $1870(5,6)$. Extensive research in the past 50 years has revealed several biological functions of Cur such as anti-proliferative, anti-inflammatory, and antioxidant properties. Recently, Cur has attracted great attention for its anticancer properties on breast, lung, prostate, and brain tumors (7). It is also known that Cur is a potent inducer of apoptotic cell death in several cancer types (8-11).

There are many studies investigating the possible apoptotic effects of Cur on various human cancer cells (7-11), but there is no study on MDR H69AR small cell lung cancer cells. In the current study, potential cyto- 
toxic and apoptotic effects of Cur were investigated on MDR H69AR human lung cancer cells. Moreover, the dominant signaling cascades and apoptotic players by Cur-induced cytotoxicity were screened by antibody array analysis.

\section{MATERIALS AND METHODS}

\section{Cells and Culture}

The human H69AR lung cancer cells utilized in the experiments were obtained from the American Type Culture Collection (ATCC, CRL-11351). H69AR cells were maintained in RPMI 1640 medium (Sigma-Aldrich, UK) with stable L-glutamine (1\%) (Sigma-Aldrich, UK), fetal bovine serum (20\%) (Sigma-Aldrich, UK) and, streptomycin (1\%) (Sigma-Aldrich, UK) in $75 \mathrm{~cm}^{2}$ flasks (Cellstar, UK). Cells were incubated in a $37^{\circ} \mathrm{C}$ incubator during the experiments.

\section{Curcumin Treatment}

Cur (Sigma-Aldrich, UK) was prepared as $1 \mathrm{mg} / \mathrm{mL}$ ethanol (EtOH) stock solution and stored for experiments at $4{ }^{\circ} \mathrm{C}$. The $\mathrm{EtOH}$ concentration in all experimental wells was lower than $0.1 \%$ and was not toxic to the cells (Figure $1 \mathrm{~A}-\mathrm{C}$ ).

\section{MTT Assay}

MTT (3-(4,5-dimethylthiazol-2-yl)-2,5-diphenyltetrazolium bromide) assay (Sigma-Aldrich, UK) was utilized to calculate the viability of cells after Cur exposure. Cells (104/well) were propagated onto 96 -well plates in a $200 \mu \mathrm{L}$ medium. After 24,48 and $72 \mathrm{~h}$ of exposure with increasing concentrations of Cur (5$100 \mu \mathrm{g} / \mathrm{mL}$ ), $20 \mu \mathrm{L}$ MTT was added to each well and incubated for an additional $4 \mathrm{~h}$ at $37^{\circ} \mathrm{C}$. After the incubation period, wells were drained and existent crystals were dissolved by DMSO (Sigma-Aldrich, UK). Conversion of MTT into formazan by mitochondrial succinate dehydrogenase and other oxidoreductases was detected via spectrophotometer $(570 \mathrm{~nm}$, Tecan Infinite 200 PRO, Switzerland) (12). The half-maximal inhibitory concentration $\left(\mathrm{IC}_{50}\right)$ was evaluated via Graphpad 5.0 software.

\section{Detection of Apoptosis via ELISA Assay}

Apoptosis was determined through the Cell Death Detection kit (Sigma Aldrich, UK). This ELISA kit detects DNA fragments in the cytoplasm of apoptotic cells. For this experiment, cells were exposed to Cur (5-100 $\mu \mathrm{g} / \mathrm{mL}$ ) for 24,48 and $72 \mathrm{~h}$. After each incubation period, cells were lysed and cytoplasmic fractions were obtained. Then, $20 \mu \mathrm{L}$ of the cytoplasmic fractions were put into a streptavidin-coated 96-well. The mixture of "anti-DNA" and "anti-histone" was added to all wells and additionally incubated for 2 h. After washing the plates, diammonium salt was added and optic densities were measured at $405 \mathrm{~nm}$ and $490 \mathrm{~nm}$ (Tecan Infinite 200 PRO, Switzerland). The fold changes in each treated well were determined as compared to untreated controls.

\section{Protein Array}

Apoptosis related proteins of H69AR cells were analyzed by an Apoptosis Antibody Array (R\&D Systems, USA). The principle of the array method involves a sandwich immunoassay, which is membrane-based (13). To optimize the exposure time, which allows us the detection of all protein spots, different exposure times were tested for each membrane ranging from $15 \mathrm{~s}$ to 15 $\mathrm{min}$. Finally, it was optimized to use for $5 \mathrm{~min}$. The proteins were detected by chemiluminescence methods involving an incubation step with an antibody (biotinylated) for $h$ and, with streptavidin (HP-conjugated) for $1 \mathrm{~h}$. Chemiluminescence imaging was conducted via UVP Biolmaging Systems. Koadaarray ${ }^{\circledR} 2.6$ software was used to quantitate the pixel intensity in each spot (14).

\section{Analysis of Protein-Protein Interaction}

Protein-protein interactions were analyzed and visualized by using the STRING database. (version 11.0; http://string.embl.de) (15).

\section{Statistical Analysis}

All statistical analyses were done by using Graphpad Prism 5.0 software (USA). For significant values, a one-way analysis of variance test (ANOVA) was utilized (16). Statistically significant data were those with a $p$ value $\leq 0.05$.

\section{RESULTS}

Cur Inhibits the Viability of H69AR Human Lung Cancer Cells The effect of Cur on the viability of H69AR cells was assessed in a concentration- and time-dependent manner. As shown in Figure 1(A-C), the viability of H69AR cells was reduced at 24, 48 and $72 \mathrm{~h}$. Reduction in the viability was also concentration-dependent at all tested time points. There were $1 \%, 73 \%$, and $96 \%$ reductions in the viability of H69AR cells exposed to 5,25 , and $100 \mu \mathrm{g} / \mathrm{mL}$ Cur respectively, as compared to control cells at $24 \mathrm{~h}(\mathrm{p}<0.05)$. There were $2 \%$, $88 \%$, and $97 \%$ reductions in the viability of H69AR cells exposed to 5,25 and $100 \mu \mathrm{g} / \mathrm{mL}$ Cur respectively, as compared to control cells at
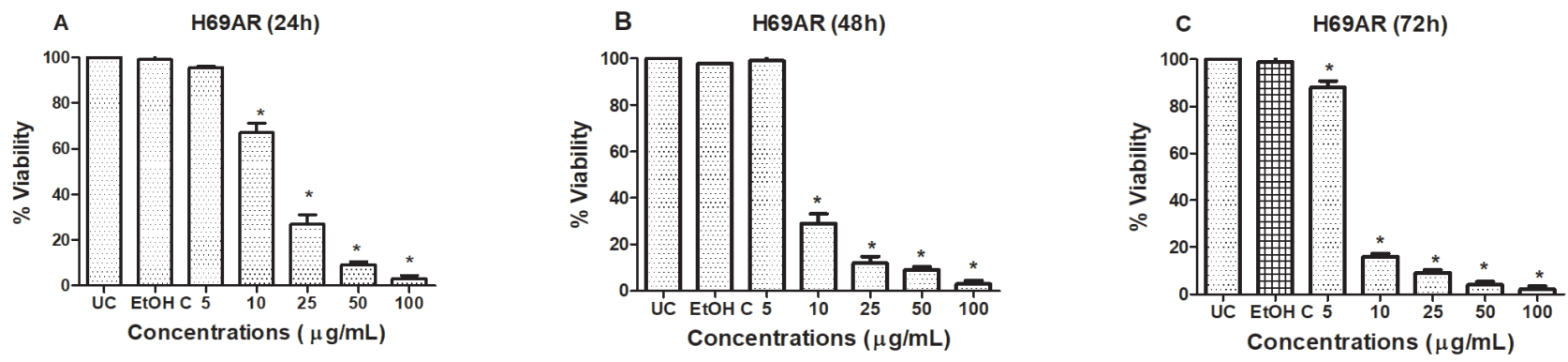

Figure 1. Concentration- and time-dependent inhibition of the viability of H69AR lung cancer cells by Cur. Cell viability was determined by the MTT assay. The results are the mean of 3 different experiments ( $\pm S D)\left({ }^{*} p<0.05\right.$ compared to untreated cells) (UC: Untreated control, EtOH C: Ethanol control). 
A

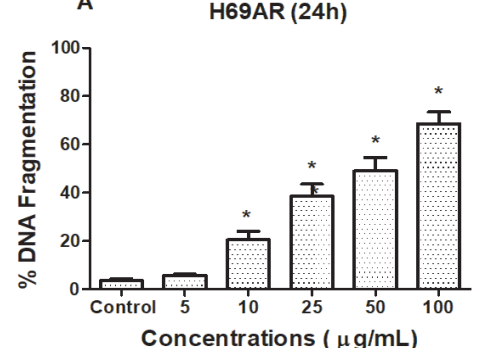

B

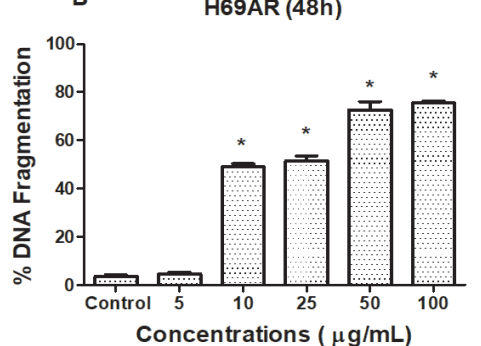

C

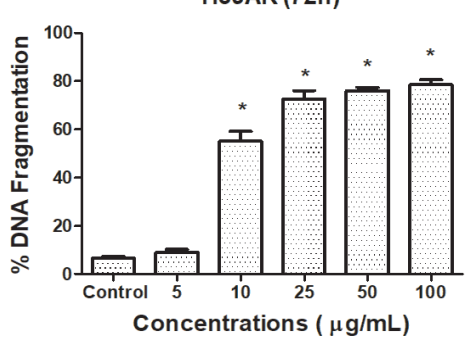

Figure 2. Induction of $\%$ of DNA fragmentation in H69AR cells in response to Cur treatment at different time points. $\left({ }^{*} \mathrm{p}<0.05\right.$ compared to untreated cells).

$48 \mathrm{~h}(\mathrm{p}<0.05)$. The most effective cytotoxic time point was accepted as $48 \mathrm{~h}$ and $\mathrm{IC}_{50}$ value of Cur in H69AR cells was $8.75 \mu \mathrm{g} / \mathrm{mL}$.

\section{Induction of Apoptotic Cell Death by Cur in H69AR Cells}

To assess the induction of apoptosis after exposure to Cur in H69AR cells, increasing concentrations of Cur were applied for $24 \mathrm{~h}, 48 \mathrm{~h}$ and $72 \mathrm{~h}$ and then apoptotic cell death was evaluated. As shown in Figure 2, Cur exposure increased the DNA fragments concentration dependently in H69AR cells $(p<0.05)$.

\section{Changes in Apoptotic Proteins by Cur in H69AR Cells}

Differences in the expression of apoptosis-related proteins were profiled by using apoptosis array in H69AR cells. Table 1 shows the changes in the expression of apoptotic proteins in H69AR cells after Cur exposure. The results revealed that pro-apoptotic proteins such as Bad, Bax, Caspase-3, TRAIL R1, TRAIL R2, FADD, Fas, SMAC/DIABLO, HMOX2 were significantly increased by 2.4-, 3.1-, 2.6-, 3.1-, 3.4-, 2.4-, 2.1-, 4.1- and 5.5-fold in H69AR cells, respectively, as compared to control cells at $48 \mathrm{~h}(\mathrm{p}<0.05)$. The anti-apoptotic proteins such as Bcl-2, ClAP-1, CLU and HIF1A were significantly decreased by 4.1-, 3.2-, 2.2- and 2.0-fold in H69AR cells, respectively, at $48 \mathrm{~h}(\mathrm{p}<0.05)$.

\section{Protein-Protein Interaction}

To analyze protein-protein interactions, the STRING database was utilized. Figure 3 indicates the protein-protein interaction generated by STRING with an average local clustering coefficient of 0.491 .

\section{DISCUSSION}

Curcumin, the active component of C. longa, has been used traditionally for centuries to treat a variety of impairments and studied on a large scale for its anti-proliferative, anti-inflammatory, and anti-oxidant effects throughout the past few decades (17-19). It has been shown in many studies that Cur has potent antiproliferative effects and exerts activity through multiple signaling mechanisms (20-22). However, in the literature, there is no study investigating the effect of Cur on H69AR small cell lung cancer cells. The H69AR cells are resistant to many chemotherapeutic drugs such as adriamycin, epirubicin, daunomycin, mitoxantrone, etoposide, vincristine and vinblastine. Therefore, H69AR is known as a multi-drug resistant lung cancer cell line (23). The results revealed that Cur inhibited the viability of H69AR cells in a concentration-dependent manner. In a study by Li et al., similar results were obtained by human lung carcinoma A549 cells (24). Cur exposure significantly inhibited A549 cell proliferation and also induced apoptosis concentration dependently. Yang et

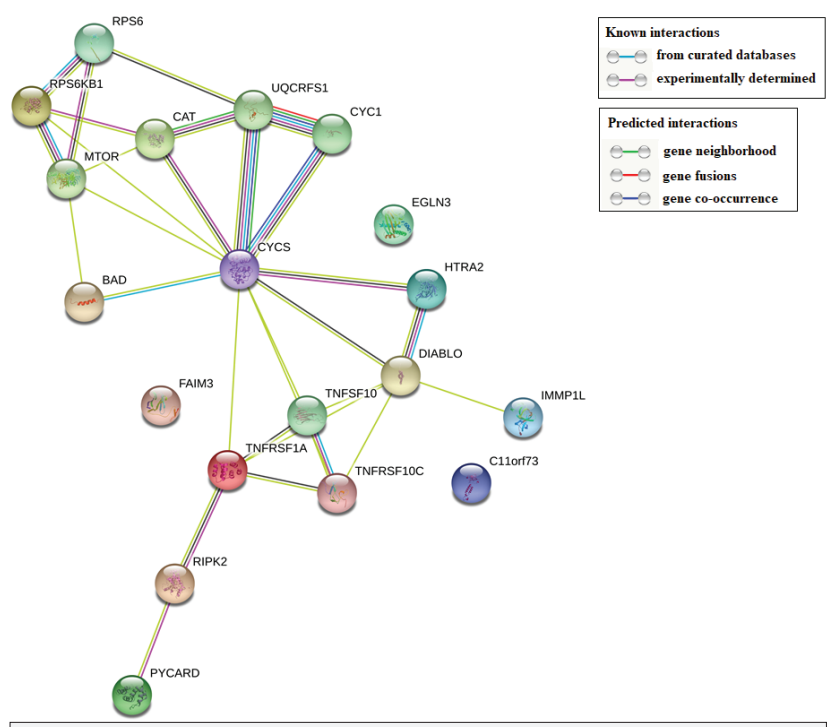

Figure 3. The protein-protein interactions map of the identified proteins. STRING database, version 11 (http://string.embl. de) was used to determine the protein-protein interactions of the proteins identified by antibody array analysis (RPS6: 40S Ribosomal Protein S6; RPS6KB1: Ribosomal Protein S6 Kinase B1; MTOR: Mechanistic Target of Rapamycin Kinase; UQCRFS1: Ubiquinol-Cytochrome C Reductase, Rieske IronSulfur Polypeptide 1, CYC1: Cytochrome C1, CYCS: Cytochrome C, EGLN3: Egl-9 Family Hypoxia Inducible Factor 3, FAIM3: Fas Apoptotic Inhibitory Molecule 3, HTRA2: HtrA Serine Peptidase 2, DIABLO: Diablo IAP-Binding Mitochondrial Protein, TNFSF10:TNF Superfamily Member 10, TNFRSF1A: TNF Receptor Superfamily Member 1A, TNFRSF10C: TNF Receptor Superfamily Member 10c, RIPK2: Receptor Interacting Serine/Threonine Kinase 2, PYCARD: Caspase Recruitment Domain-Containing Protein 5, IMMP1L: Inner Mitochondrial Membrane Peptidase Subunit 1, C11orf73: Heat Shock Protein Nuclear Import Factor Hikeshi, CLUL1: Clusterin Like 1, RFWD2: COP1 E3 Ubiquitin Ligase). 
Table 1. Changes in apoptosis related proteins in H69AR cells after exposure to curcumin $(8.75 \mu \mathrm{g} / \mathrm{mL})$ for $48 \mathrm{~h}$. The results are the mean of two independent experiments ( \pm SD) (ND: not detectable, Ns: non-significant changes).

\section{H69AR}

\begin{tabular}{|c|c|c|c|}
\hline Symbol & Protein name & Up/down-regulation & Fold change \\
\hline Bad & BCL2 Associated Agonist Of Cell Death & $\uparrow$ & $2.4 \pm 0.2$ \\
\hline Bax & BCL2 Associated X Protein & $\uparrow$ & $3.1 \pm 2.8$ \\
\hline $\mathrm{Bcl}-2$ & BCL2 Apoptosis Regulator & $\downarrow$ & $4.1 \pm 2.4$ \\
\hline $\mathrm{BCl}-\mathrm{x}$ & Bcl-2-Like Protein 1 & $\leftrightarrow$ & Ns \\
\hline Pro-Caspase-3 & Pro- apoptosis-Related Cysteine Peptidase & $\downarrow$ & $2.0 \pm 0.8$ \\
\hline Cleaved Caspase-3 & Cleaved apoptosis-Related Cysteine Peptidase & $\uparrow$ & $2.6 \pm 1.2$ \\
\hline Cat & Catalase & $\uparrow$ & $2.1 \pm 2.3$ \\
\hline CIAP-1 & Apoptosis Inhibitor 1 & $\downarrow$ & $3.2 \pm 0.2$ \\
\hline CIAP-2 & Apoptosis Inhibitor 2 & $\leftrightarrow$ & Ns \\
\hline CLSPN & Claspin & $\leftrightarrow$ & Ns \\
\hline CLU & Clusterin & $\downarrow$ & $2.2 \pm 3.0$ \\
\hline CYCS & Cytochrome c & $\uparrow$ & $4.2 \pm 2.8$ \\
\hline TRAIL R1/DR4 & Death receptor 4 & $\uparrow$ & $3.1 \pm 2.6$ \\
\hline TRAIL R2/DR5 & Death receptor 5 & $\uparrow$ & $3.4 \pm 0.8$ \\
\hline FADD & Fas Associated via Death Domain & $\uparrow$ & $2.4 \pm 2.2$ \\
\hline Fas/TNFRSF6/CD95 & Fas Cell Surface Death Receptor & $\uparrow$ & $2.1 \pm 0.4$ \\
\hline HIF-1A & Hypoxia Inducible Factor 1 Subunit Alpha & $\downarrow$ & $2.0 \pm 0.2$ \\
\hline HO-1/HMOX1/HSP32 & Heme Oxygenase 1 & $\uparrow$ & $2.5 \pm 0.8$ \\
\hline $\mathrm{HO}-2 / \mathrm{HMOX} 2$ & Heme Oxygenase 2 & $\uparrow$ & $5.2 \pm 3.8$ \\
\hline HSP27 & Heat Shock 27 KDa Protein 1 & $\uparrow$ & $2.8 \pm 0.4$ \\
\hline HSP60 & Heat Shock 60kDa Protein 1 & $\leftrightarrow$ & Ns \\
\hline HSP70 & Heat Shock 70 KDa Protein 4 & $\uparrow$ & $2.2 \pm 0.4$ \\
\hline HTRA2/Omi & HtrA Serine Peptidase 2 & $\uparrow$ & $3.6 \pm 2.6$ \\
\hline Livin & Baculoviral IAP Repeat Containing 7 & $\leftrightarrow$ & Ns \\
\hline PON2 & Paraoxonase 2 & $\leftrightarrow$ & Ns \\
\hline $\mathrm{p} 21 / \mathrm{CIP} 1 / \mathrm{CDKN} 1 \mathrm{~A}$ & Cyclin Dependent Kinase Inhibitor $1 \mathrm{~A}$ & $\leftrightarrow$ & ND \\
\hline p27/Kip1 & Cyclin Dependent Kinase Inhibitor 1B & $\leftrightarrow$ & ND \\
\hline Phospho-p53 (S15) & Tumor Protein P53 (Phospho- Ser15) & $\uparrow$ & $3.2 \pm 1.2$ \\
\hline Phospho-p53 (S46) & Tumor Protein P53 (Phospho- Ser46) & $\uparrow$ & $3.6 \pm 2.4$ \\
\hline Phospho-p53 (S392) & Tumor Protein P53 (Phospho- Ser392) & $\uparrow$ & $2.8 \pm 1.2$ \\
\hline Phospho-Rad17 (S635) & Cell Cycle Checkpoint Protein RAD17 (Phospho-Ser635) & $\leftrightarrow$ & ND \\
\hline SMAC/Diablo & Diablo IAP-Binding Mitochondrial Protein & $\uparrow$ & $4.1 \pm 0.8$ \\
\hline Survivin & Baculoviral IAP Repeat Containing 5 & $\leftrightarrow$ & ND \\
\hline TNF RI/TNFRSF1A & TNF Receptor Superfamily Member $1 \mathrm{~A}$ & $\leftrightarrow$ & $\mathrm{Ns}$ \\
\hline XIAP & X-Linked Inhibitor of Apoptosis & $\leftrightarrow$ & Ns \\
\hline
\end{tabular}

al. also investigated the cytotoxic effect of curcumin on $\mathrm{NCl}-\mathrm{H} 446$ cells and determined the $\mathrm{IC}_{50}$ value as $15 \mu \mathrm{M}$ (25). In a study by Jin et al., the apoptotic effect of Cur was investigated on A549 cells at 10-40 $\mu \mathrm{M}$ concentrations at $24 \mathrm{~h}$ and showed the apoptotic cell death ascended by the increasing concentrations, as compared to the control cells (26). Further, in the current study, apoptotic effect of Cur was also evaluated in H69AR cells by flow cytometer and found that Cur induced apoptosis at $48 \mathrm{~h}$.

To explore the potential molecular mechanisms responsible for the apoptotic activity, an antibody protein array that provides the simultaneous evaluation of 35 apoptosis-associated proteins was used. Apoptosis is a programmed cell death characterized by chromatin condensation, phosphatidylserine externalization, DNA fragmentation and apoptotic bodies which can be activated via internal or external signals (27). Apoptosis that has been initiated by external signals such as tumor necrosis factor receptor (TNF), TNF-related apoptosis-inducing ligand receptor 1 (TRAIL) and Fas cell surface death receptor (Fas) is called the extrinsic apoptotic pathway. However, the intrinsic (mitochondrial) apoptotic pathway is initiated internally and includes $\mathrm{BCl}-2$ family proteins (27-29). The Bcl-2 family proteins containing both 
pro-apoptotic and anti-apoptotic members are the main regulators of intrinsic apoptosis. Pro-apoptotic proteins such as Bad and Bak, which are essential for mitochondrial permeabilization, are activators of apoptosis whereas anti-apoptotic members such as $\mathrm{BCl}-2, \mathrm{BCl}-\mathrm{xL}$ and $\mathrm{Mcl}-1$ inhibit apoptotic cell death (28). Results revealed an increase in the pro-apoptotic proteins belonging to the intrinsic apoptotic pathways such as Bad, Bax and a decrease in $\mathrm{BCl}-2$. Zhu et al. showed that protein expression of Bax was induced, while $\mathrm{Bcl}-2$ was reduced indicating the induction of apoptosis by the high dosing groups of Cur in pancreatic cancer cells (30). Yang et al., also demonstrated changes in $\mathrm{Bax}, \mathrm{Bcl}-2$ and Bcl-xL proteins in $\mathrm{NCl}-\mathrm{H} 446$ cells by $15 \mu \mathrm{M}$ Cur for $48 \mathrm{~h}$ (25). Cytochrome $c$ release from the mitochondria is essential for the activation of caspase- 9 and as a result, activation of apoptosis (27). In the same study, authors also stated that Cur induced cytochrome c, caspase- 9 and caspase- 3 levels. In the current study, array results support the findings of Yang et al. in the case of induction in cytochrome c release and increase in caspase-3 levels (25). However, the array results of the current study do not support some of the findings of Yang et al. They reported that Cur did not cause any change in the expression of FAS and TRAIL apoptotic receptors and stated that Cur did not induce death receptor-mediated pathways (25). In the current study, Cur induced the main death receptors (TRAIL R1, TRAIL R2, FADD and Fas) indicating the induction of death receptor-mediated apoptotic pathway in H69AR cells. Reactive oxygen species (ROS) and mitochondria both play a vital role in the activation of apoptosis. Cytochrome $c$ release is known to be mainly regulated via ROS (29). In many studies, the induction of the ROS-mediated mitochondrial pathway in Cur-treated cells has been demonstrated $(31,32)$. Results of Kuttikrishnane et al. revealed the activation of the intrinsic apoptotic pathway via the generation of ROS in acute lymphoblastic leukemia cells by Cur treatment (33). Similarly, according to array results, Cur induced the proteins involved in ROS-mediated apoptotic pathways such as HIF-1A, HSP27 and HSP70. Here, changes in apoptotic proteins by Cur treatment were investigated by using a protein array method, but this needs to be verified by other methods such as western blotting or qPCR.

To investigate the possible protein-protein interactions of the proteins obtained from array data, bioinformatics STRING analysis was carried out. Combining the protein array data with protein interactome data allows us to investigate the network of proteins that Cur is probably interacting with. The network interaction map revealed complex protein-protein interactions that include direct and indirect functional protein connections. Of these proteins, Ribosomal Protein S6 (RPS6) is a cytoplasmic ribosomal protein and one of the components of the $40 \mathrm{~S}$ ribosomal subunit. It is the downstream substrate of PI3K/Akt/ mTOR/p70S6 kinase pathways and is phosphorylated by several kinases such as Ribosomal Protein S6 Kinase B1 (RPS6KB1) (34). In the literature, it has been shown that Cur inhibits the mTOR pathway via decreasing the phosphorylation of RPS6 leading to blocking the proliferation of human colorectal cancer cells, malignant glioma cells and intestinal epithelial cells (35-37). According to the map, Cur could induce apoptosis in H69AR cells by inhibiting mTOR pathway and phosphorylation of RPS6.
The interaction map also revealed the interaction of TNFSF10 (TRAIL) with Receptor-interacting serine threonine kinase 2 (RIPK2) which is one of the members of the serine/threonine protein kinase family. It acts by binding to the inhibitor of apoptosis proteins (AIPs) such as XIAP, cIAP-1 and CIAP-2. It was shown by the array data that Cur induced both TRAIL R1 and TRAIL R2 and subsequently inhibited CIAP-1, which leads to enhancement of the apoptotic pathway. The STRING data implies the possible interaction of TNFSF10 with RIPK2 and Caspase Recruitment Domain-Containing Protein 5 (PYCARD) after Cur treatment in H69AR cells. PYCARD functions as a key mediator in the activation of the mitochondrial apoptotic pathway and also regulates mitochondrial translocation of BAX and activates initiator caspases, which were found to be increased after Cur treatment in H69AR cells (38).

Although the present findings are notable, there are limitations to the study. The effects of Cur on human MDR small cell lung cancer cells were only detected on H69AR cells. The study should be expanded by using other NSCLC cell lines.

\section{CONCLUSION}

Collectively, the novel findings of this study from array data and STRING database suggested that Cur inhibited cell viability and induced apoptotic cell death in human H69AR cells via mediating several proteins involved in both extrinsic and intrinsic apoptotic pathways. By expanding our knowledge of the heterogeneous, biological behavior of the MDR H69AR cells, novel treatment approaches can be developed for the treatment of MDR small cell lung cancer.

Peer-review: Externally peer-reviewed.

Author Contributions: Conception/Design of study: S.i.; Data Acquisition: S.I.; Data Analysis/Interpretation: S.I.; Drafting Manuscript: S.I.; Critical Revision of Manuscript: S.I.; Final Approval and Accountability: S.i.

Conflict of Interest: The authors declare that they have no conflicts of interest to disclose.

Financial Disclosure: There are no funders to report for this submission.

\section{REFERENCES}

1. Molina JR, Adjei AA, Jett JR. Advances in chemotherapy of nonsmall cell lung cancer. Chest 2006; 130: 1211-9.

2. Pfister DG, Johnson DH, Azzoli CG, Sause W, Smith TJ, Baker JrS, et al. American Society of Clinical Oncology treatment of unresectable non-small-cell lung cancer guideline: Update 2003. J Clin Oncol 2004; 22: 330-53.

3. Sosa Iglesias V, Giuranno L, Dubois LJ, Theys J, Vooijs M. Drug resistance in non-small cell lung cancer: a potential for NOTCH targeting? Front Oncol 2018; 8: 267.

4. Alibeiki F, Jafari N, Karimi M, Peeri Dogaheh H. Potent anti-cancer effects of less polar Curcumin analogues on gastric adenocarcinoma and esophageal squamous cell carcinoma cells. Sci Rep 2017; 7: 1-9. 
5. Aggarwal BB, Surh YJ, Shishodia S. The molecular targets and therapeutic uses of curcumin in health and disease. $1^{\text {st }}$ edition. New York: Springer Science \& Business Media; 2007.

6. Priyadarsini KI. The chemistry of curcumin: From extraction to therapeutic agent. Molecules 2014; 19: 20091-12.

7. Anand P, Sundaram C, Jhurani S, Kunnumakkara AB, Aggarwal BB. Curcumin and cancer: An "old-age" disease with an "age-old" solution. Cancer Lett 2008; 267: 133-64.

8. Dorai T, Cao YC, Dorai B, Buttyan R, Katz AE. Therapeutic potential of curcumin in human prostate cancer. III. Curcumin inhibits proliferation, induces apoptosis, and inhibits angiogenesis of LNCaP prostate cancer cells in vivo. Prostate 2001; 47: 293-303.

9. Mukhopadhyay A, Bueso-Ramos C, Chatterjee D, Pantazis P, Aggarwal BB. Curcumin downregulates cell survival mechanisms in human prostate cancer cell lines. Oncogene 2001; 20: 7597-7609.

10. Liu Q, Loo WTY, Sze SCW, Tong Y. Curcumin inhibits cell proliferation of MDA-MB-231 and BT-483 breast cancer cells mediated by down-regulation of NFB, cyclinD and MMP-1 transcription. Phytomedicine 2009; 16: 916-22.

11. Moragoda L, Jaszewski R, Majumdar AP. Curcumin induced modulation of cell cycle and apoptosis in gastric and colon cancer cells. Anticancer Res 2001; 21: 873-8.

12. Atmaca $\mathrm{H}$, Uzunoglu S. Anti-angiogenic effects of trabectedin (Yondelis; ET-743) on human breast cancer cells. Eur Cytokine Netw 2014; 25: 1-7.

13. Karaca B, Kucukzeybek Y, Gorumlu G, Erten C, Gul MK, Cengiz E et al. Profiling of angiogenic cytokines produced by hormone-and drug-refractory prostate cancer cell lines, PC-3 and DU-145 before and after treatment with gossypol. Eur Cytokine Netw 2008; 19: 176-84.

14. Erten C, Karaca B, Kucukzeybek Y, Gorumlu G, Cengiz E, Gul MK, et al. Regulation of growth factors in hormone-and drug-resistant prostate cancer cells by synergistic combination of docetaxel and octreotide. BJU Int 2009; 104, 107-14.

15. Von Mering C, Jensen LJ, Snel B, Hooper SD, Krupp M, Foglierini M, et al. STRING: known and predicted protein-protein associations, integrated and transferred across organisms. Nucleic Acids Res 2005; 33: 433-7.

16. Scheff SW. Fundamental statistical principles for the neurobiologist: A survival guide. $1^{\text {st }}$ edition. New York : Academic Press; 2016.

17. Boroumand N, Samarghandian S, Hashemy SI. Immunomodulatory, anti-inflammatory, and antioxidant effects of curcumin. J Herbmed Pharmacol 2018; 7: 211-9.

18. Jurenka JS. Anti-inflammatory properties of curcumin, a major constituent of Curcuma longa: a review of preclinical and clinical research. Altern Med Rev 2009; 14: 141-53.

19. Hewlings SJ, Kalman DS. Curcumin: a review of its' effects on human health. Foods 2017; 6: 92.

20. Tomeh MA, Hadianamrei R, Zhao X. A review of curcumin and its derivatives as anticancer agents. Int J Mol Sci 2019; 20: 1033.

21. Allegra A, Innao V, Russo S, Gerace D, Alonci A, Musolino C. Anticancer activity of curcumin and its analogues: preclinical and clinical studies. Cancer Invest 2017; 35: 1-22.

22. Vallianou NG, Evangelopoulos A, Schizas N, Kazazis C. Potential anticancer properties and mechanisms of action of curcumin. Anticancer Res 2015; 35: 645-51.
23. Mirski SE Gerlach JH, Cole SP. Multidrug resistance in a human small cell lung cancer cell line selected in adriamycin. Cancer Res 1987; 47: 2594-8.

24. Li P, Qin H, Li X. The effect of curcumin on the apoptosis of lung cancer cells by regulating DJ-1-PTEN/PI3K/AKT signaling. Int J Clin Exp Med 2019; 12: 8739-45.

25. Yang CL, Ma YG, Xue YX, Liu YY, Xie H, Qiu GR. Curcumin induces small cell lung cancer $\mathrm{NCl}-\mathrm{H} 446$ cell apoptosis via the reactive oxygen species mediated mitochondrial pathway and not the cell death receptor pathway. DNA Cell Biol 2012; 31: 139-50.

26. Jin H, Qiao F, Wang Y, Xu Y, Shang Y. Curcumin inhibits cell proliferation and induces apoptosis of human non-small cell lung cancer cells through the upregulation of miR-192-5p and suppression of PI3K/Akt signaling pathway. Oncol Rep 2015; 34: 2782-9.

27. Jin Z, El-Deiry WS. Overview of cell death signaling pathways. Cancer Biol Ther 2005; 4: 147-71.

28. Giam M, Huang DCS. Bouillet, P. BH3-only proteins and their roles in programmed cell death. Oncogene 2008; 27: 128-36.

29. Zuo L, Zhou T, Pannell BK, Ziegler AC, Best TM. Biological and physiological role of reactive oxygen species-the good, the bad and the ugly. Acta Physiol 2015; 214: 329-48.

30. Zhu Y, Bu S. Curcumin induces autophagy, apoptosis, and cell cycle arrest in human pancreatic cancer cells. Evid Based Complementary Altern Med 2017; 2017.

31. Larasati YA, Yoneda-Kato N, Nakamae I, Yokoyama T, Meiyanto E, Kato JY. Curcumin targets multiple enzymes involved in the ROS metabolic pathway to suppress tumor cell growth. Sci Rep 2018; 8: 1-13.

32. Chen $Q$, Wang Y, Xu K, Lu G, Ying Z, Wu L, et al. Curcumin induces apoptosis in human lung adenocarcinoma A549 cells through a reactive oxygen species-dependent mitochondrial signaling pathway. Oncol Rep 2010; 23: 397-403.

33. Kuttikrishnan S, Siveen KS, Prabhu KS, Khan AQ, Ahmed El, Akhtar $S$, et al. Curcumin induces apoptotic cell death via inhibition of $\mathrm{PI3-Kinase/AKT} \mathrm{pathway} \mathrm{in} \mathrm{B-precursor} \mathrm{acute} \mathrm{lymphoblastic} \mathrm{leu-}$ kemia. Front Oncol 2019: 9.

34. Lu T, Zhu Z, Wu J, She H, Han R, Xu H, et al. DRAM1 regulates autophagy and cell proliferation via inhibition of the phosphoinositide 3-kinase-Akt-mTOR-ribosomal protein S6 pathway. Cell Commun Signal 2019; 17: 28.

35. Johnson SM, Gulhati P, Arrieta I, Wang X, Uchida T, Gao T, et al. Curcumin inhibits proliferation of colorectal carcinoma by modulating Akt/mTOR signaling. Anticancer Res 2009; 29: 3185-90.

36. Shinojima N, Yokoyama T, Kondo Y, Kondo, S. Roles of the Akt/ $\mathrm{mTOR} / \mathrm{p} 70 \mathrm{S6K}$ and ERK1/2 signaling pathways in curcumin-induced autophagy. Autophagy 2007; 3: 635-7.

37. Moreau R, Kaur H. Curcumin and piperine inhibit mTORC1 signaling in intestinal epithelial cells. The FASEB Journal 2017; 31: 135-8.

38. Srinivasula SM, Poyet JL, Razmara M, Datta P, Zhang Z, Alnemri ES. The PYRIN-CARD protein ASC is an activating adaptor for caspase-1. J Biol Chem 2002; 277: 21119-22. 